\title{
Blocking Artifacts Suppression in Block-Coded Images Using Overcomplete Wavelet Representation
}

\author{
Alan W.-C. Liew, Member, IEEE, and Hong Yan, Senior Member, IEEE
}

\begin{abstract}
It is well known that at low-bit-rate block discrete cosine transform compressed image exhibits visually annoying blocking and ringing artifacts. In this paper, we propose a noniterative, wavelet-based deblocking algorithm to reduce both types of artifacts. The algorithm exploits the fact that block discontinuities are constrained by the dc quantization interval of the quantization table, as well as the behavior of wavelet modulus maxima evolution across wavelet scales to derive appropriate threshold maps at different wavelet scales. Since ringing artifacts occur near strong edges, which can be located either along block boundaries or within blocks, suppression of block discontinuities does not always reduce ringing artifacts. By exploiting the behavior of ringing artifacts in the wavelet domain, we propose a simple yet effective method for the suppression of such artifacts. The proposed algorithm can suppress both block discontinuities and ringing artifacts effectively while preserving true edges and textural information. Simulation results and extensive comparative study with both iterative and noniterative methods reported in the literature have shown the effectiveness of our algorithm.
\end{abstract}

Index Terms-Block discrete cosine transform (BDCT), blocking artifacts, overcomplete wavelet, wavelet deblocking.

\section{INTRODUCTION}

B LOCK-BASED discrete cosine transform (BDCT) [1] has been widely used in image and video compression. In BDCT coding, an image is first divided into $8 \times 8$ nonoverlapping blocks. Each block is then transformed using the DCT, followed by quantization and variable length coding. However, at low bit rates, the coarse quantization of the DCT coefficients causes artificial discontinuities to be created along the block boundaries in the compressed image. This unpleasant visible degradation is due to the negligence of correlation among adjacent blocks, i.e., two low-frequency DCT coefficients in adjacent blocks, which are similar in value, are quantized into different quantization bins. The coarse quantization also causes high-frequency DCT coefficients to be truncated and produces ringing artifacts near strong edges.

Manuscript received November 18, 2000; revised August 18, 2003. This work was supported by the Research Grant Council of Hong Kong under Project CityU 1088/00E and CityU Grant 9380018 . This paper was recommended by Associate Editor O. K. Al-Shaykh.

A. W.-C. Liew is with the Department of Computer Engineering \& Information Technology, City University of Hong Kong, Kowloon Tong, Hong Kong (e-mail: itwcliew@cityu.edu.hk).

H. Yan is with the Department of Computer Engineering \& Information Technology, City University of Hong Kong, Kowloon Tong, Hong Kong, and also with the School of Electrical and Information Engineering, University of Sydney, NSW 2006, Australia.

Digital Object Identifier 10.1109/TCSVT.2004.825555
Many postprocessing methods have been proposed to remove the blocking artifacts [2]-[12], [15], [16], [20]-[27]. The projection onto convex sets (POCS) algorithm [4]-[6], [11], [24] deblocks images by iterating the projections onto the quantization constraint set and the image smoothness constraint set until convergence. A major concern with the POCS method is the computational complexity, but it can usually produce good results. In [7]-[10], noniterative, spatially adaptive postfiltering is proposed for image deblocking. However, at low bit rates, block discontinuities cannot be completely eliminated by spatially adaptive postfiltering. In addition, the deblocked image may not satisfy the quantization constraint imposed by the encoder. In [15] and [16], image deblocking based on the wavelet soft-thresholding concept of [13] and [14] is proposed. The multiple resolution property and the spatial-frequency localization of the wavelet allow the meaningful structures in an image to be analyzed at different scales. The wavelet soft-thresholding is effective in removing noise while preserving edges. However, the blocking artifacts problem cannot be solved simply by denoising, since the blocking artifacts are structured and concentrate mainly along block boundaries. Furthermore, texture regions may be oversmoothed by this method as they behave similarly as noise. Recently, image deblocking algorithms using the overcomplete wavelet representation [17], [18] has been proposed [20]-[23]. The overcomplete wavelet representation enables a multiscale edge analysis and is useful for edge-based image compression [19]. In [20], the Lipschitz regularity of the wavelet modulus maxima (WTMM) is used for texture/nontexture region classification. Then, the WTMM in the nontexture region are processed and the deblocked image is recovered using POCS. In contrast, the algorithms in [21]-[23] work directly on the wavelet coefficients and obtain the deblocked image by performing an inverse transform on the processed coefficients.

In this paper, we describe a novel wavelet-based deblocking algorithm for block artifacts suppression. Based on a theoretical analysis of the blocking artifacts, the proposed algorithm is able to take into account the statistical characteristic of block discontinuities, as well as the behavior of wavelet coefficients across scales for different image features, to suppress both the blocking and ringing artifacts. The paper is organized as follows. Section II gives an overview of the overcomplete wavelet representation scheme used in our algorithm. Section III carries out an analysis of block discontinuities due to BDCT compression. In particular, we show that the block discontinuities are constrained by the quantization step size of the dc coefficient and the distribution of the absolute pixel differences across 
block boundaries changes according to the quantization table used. Our deblocking algorithm is described in detail in Section IV. Simulation results and comparative studies with several iterative and noniterative algorithms are presented in Section V. Finally, Section VI concludes the paper.

\section{OVERCOMPLETE WAVELET REPRESENTATION}

The overcomplete two-dimensional (2-D) dyadic wavelet representation [17], [18] of an image $f \in L^{2}\left(R^{2}\right)$ is given by $\left\{\left(W_{2^{j}}^{1} f\right)_{1 \leq j \leq J},\left(W_{2^{j}}^{2} f\right)_{1 \leq j \leq J}, S_{2^{J}} f\right\}$. Each component is obtained by the convolution of $f(x, y)$ with the scaling function $\phi$ and the wavelets $\psi$ as follows:

$$
\begin{aligned}
W_{2^{j}}^{1} f(x, y) & =f * \psi_{2^{j}}^{1}(x, y) \\
W_{2^{j}}^{2} f(x, y) & =f * \psi_{2^{j}}^{2}(x, y) \\
S_{2^{J}} f(x, y) & =f * \phi_{2^{J}}(x, y) .
\end{aligned}
$$

For a cubic spline wavelet, the 2-D dyadic wavelet representation of an image gives the gradient of the image at multiples of dyadic scales, i.e., $W_{2^{j}}^{1} f(x, y)$ and $W_{2^{j}}^{2} f(x, y)$ gives the vertical and horizontal gradient at scale $j$, respectively. The overcomplete dyadic wavelet transform holds the size of the transformed image the same as the original image, without the downsampling operation. This scheme makes the position of the block boundaries in each scale consistent with those in the spatial domain and facilitates the removal of block discontinuities at those positions.

For discrete image $f(m, n)$, the dyadic wavelet transform is implemented by using digital filters [17]. The 2-D convolution is implemented as two separable one-dimensional (1-D) convolutions, first along the row direction and then along the column direction. Let the original image $f$ be given by $S_{2^{0}} f=f$. Let $f *(H, L)$ denote the convolution of image $f$ along the row direction with 1-D filter $H$, followed by the convolution of the result along the column direction with 1-D filter $L$. Then, the wavelet transform at the $j$ th scale is the decomposition of $S_{2^{j-1}} f$ into the detailed images $W_{2^{j}}^{1} f$ and $W_{2^{j}}^{2} f$ and the coarse image $S_{2^{j}} f$ as follows:

$$
\begin{aligned}
W_{2^{j}}^{1} f & =S_{2^{j-1}} f *\left(G_{j-1}, D\right) \\
W_{2^{j}}^{2} f & =S_{2^{j-1}} f *\left(D, G_{j-1}\right) \\
S_{2^{j}} f & =S_{2^{j-1}} f *\left(H_{j-1}, H_{j-1}\right)
\end{aligned}
$$

where $G_{j}$ and $H_{j}$ are obtained by inserting $2^{j}-1$ zeros between consecutive coefficients of the highpass filter $G$ and the lowpass filter $H$, respectively, and $D$ is the Dirac filter with an impulse response equal to one at the origin and zero elsewhere. The original image can be reconstructed by cascading the following operation from scale $J$ up to the original scale zero:

$$
\begin{gathered}
S_{2^{j-1}} f=W_{2^{j}}^{1} f *\left(K_{j-1}, L_{j-1}\right)+W_{2^{j}}^{2} f *\left(L_{j-1}, K_{j-1}\right) \\
+S_{2^{j}} f *\left(\bar{H}_{j-1}, \bar{H}_{j-1}\right)
\end{gathered}
$$

where $\bar{H}$ is the time reverse of $H$, and $K$ and $L$ are the highpass reconstruction filters.

\section{ANALYSIS OF BLOCK DiscontinUITY}

Let $f(m, n)$ and $v(m, n)$ be the original image in the spatial and the DCT domains, respectively. After quantization, let $\hat{f}(m, n)$ and $\hat{v}(m, n)$ be the reconstructed image in the spatial and DCT domains, respectively. The vertical $v b$ and horizontal $h b$ block differences, for $8 \times 8$ blocks, can be written as

$$
\begin{aligned}
& v b=\left\{\hat{f}_{a, b+1}(i, 0)-\hat{f}_{a, b}(i, 7)\right\}_{0 \leq i \leq 7,0 \leq a<\frac{M}{8}, 0 \leq b<\frac{N}{8}-1} \\
& h b=\left\{\hat{f}_{a+1, b}(0, j)-\hat{f}_{a, b}(7, j)\right\}_{0 \leq j \leq 7,0 \leq a<\frac{M}{8}-1,0 \leq b<\frac{N}{8}}
\end{aligned}
$$

where the subscripts $a$ and $b$ are the block indices and $i$ and $j$ here refer to the block-centered pixel indices. It can be shown that the vertical block difference (similarly, the horizontal block difference) at row $i$ in block $(a, b)$ is related to the DCT coefficients by

$$
\begin{aligned}
v b_{a, b}(i)= & \hat{f}_{a, b+1}(i, 0)-\hat{f}_{a, b}(i, 7) \\
= & \frac{1}{4} \sum_{k=0}^{7} \sum_{l=0}^{7} C_{k} C_{l}\left[\hat{v}_{a, b+1}(k, l)-(-1)^{l} \hat{v}_{a, b}(k, l)\right] \\
& \times \cos \frac{(2 i+1) k \pi}{16} \cos \frac{l \pi}{16}
\end{aligned}
$$

where $C_{n}=1 / \sqrt{2}$ when $n=0$ and 1 otherwise. As the low-frequency coefficients in the DCT domain are usually much larger than the high-frequency coefficients, (5) is dominated by the term $k=l=0$. Therefore, (5) can be approximated by

$$
\begin{aligned}
v b_{a, b}(i) & =\hat{f}_{a, b+1}(i, 0)-\hat{f}_{a, b}(i, 7) \\
& \approx \frac{1}{4} C_{0}^{2}\left[\hat{v}_{a, b+1}(0,0)-\hat{v}_{a, b}(0,0)\right] \\
& =\frac{1}{8}\left[Q\left\{v_{a, b+1}(0,0)\right\}-Q\left\{v_{a, b}(0,0)\right\}\right]
\end{aligned}
$$

where $Q$ denotes the quantization operation. As can be seen from the above analysis, the magnitude of the mismatch between neighboring blocks is constrained by the dc quantization step size. Thus, at very low bit rates where the quantization step size is large, the block discontinuities are particularly serious and visually annoying. Fig. 1 shows the normalized histogram plots of the absolute vertical block differences $|v b|$ for (a) the original $512 \times 512$ "Lena" image and the BDCT compressed images using quantization table: (b) Q1, (c) Q2, and (d) Q3, shown in the Appendix. Quantization causes the appearance of a peak in the histogram. In accordance with (6), the peak occurs at one eighth the dc quantization interval of the corresponding quantization table, i.e., at 6.25 for Fig. 1(b), at 10.75 for Fig. 1(c), and at 13.75 for Fig. 1(d).

Wavelets can be used to characterize the local regularity of a signal. The evolution of the wavelet modulus maxima across the scale can be used to estimate the local Lipschitz regularity of the signal [18]. The Lipschitz exponent $\alpha$ at a point $t_{0}$ of a function $f$ is related to the wavelet modulus maxima $M_{2^{j}} f$ of this function at scale $2^{j}$ by

$$
\left|M_{2^{j}} f\right| \leq A\left(2^{j}\right)^{\alpha}
$$

where $A$ is a constant. A function $f$ that is continuously differentiable at a point is Lipschitz 1 at this point. If the derivative of $f$ is bounded but discontinuous at $t_{0}, f$ is still Lipschitz 1 at this 


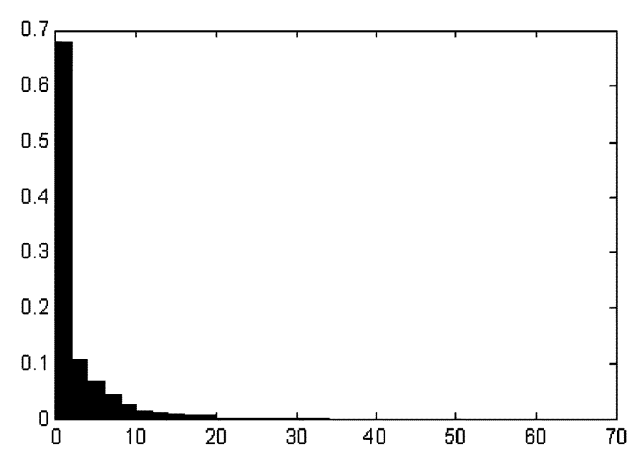

(a)

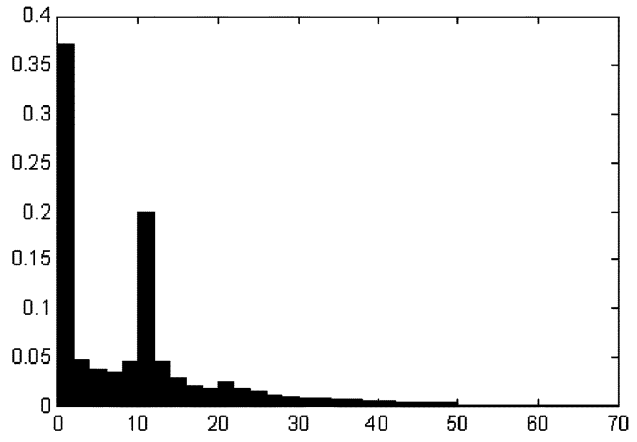

(c)

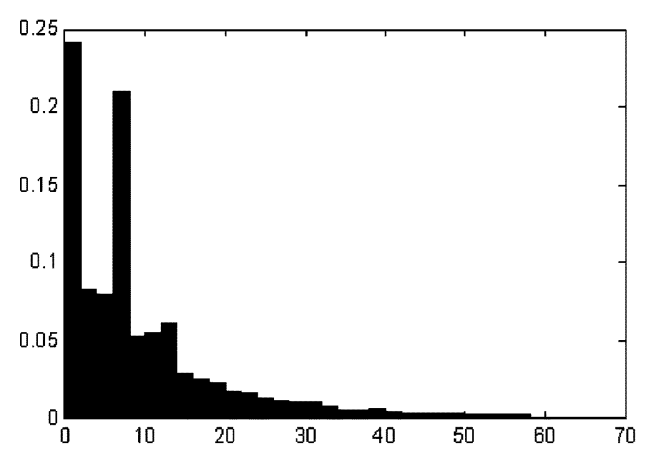

(b)

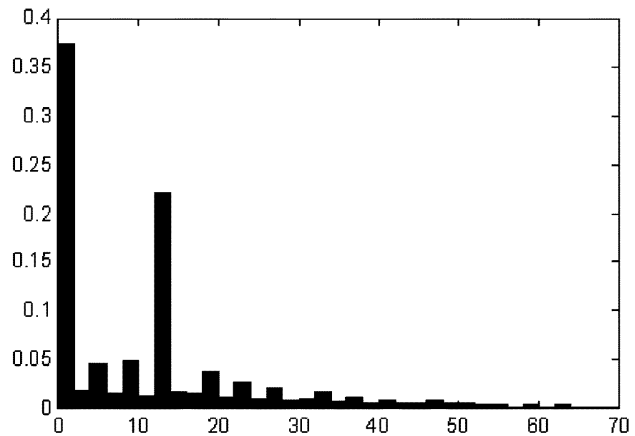

(d)

Fig. 1. Normalized histogram plots of the absolute vertical block differences $|v b|$ for (a) the original $512 \times 512$ "Lena" image, and the BDCT compressed images using quantization table: (b) Q1, (c) Q2, and (d) Q3, in the Appendix.

point and the function is not singular at $t_{0}$. A function is said to be singular at $t_{0}$ if it is not Lipschitz 1 at $t_{0}$. A step discontinuity, for example, has a Lipschitz regularity of zero. The BDCT produces block discontinuities along block boundaries. The coarse quantization also produces ringing artifacts near strong edges. The ringing artifacts can be viewed as an impulse-like structure in the image. Since $\alpha=0$ for a step discontinuity and $\alpha=-1$ for an impulse, according to (7), the wavelet modulus maxima for a step discontinuity remains constant across the scale and the wavelet modulus maxima for an impulse decays by $2^{-j}$ across the scale. Fig. 2 shows the behavior of the wavelet transform of different signal feature across scales. The wavelet coefficients at different scales are scaled to compensate for discretization effect, such that the wavelet modulus maxima of a step edge have the same amplitude at all scales as they should in a continuous model. The Lipschitz exponent for the features, from left to right, is $-1,0$, and 1 , respectively. One can see that the evolution of the wavelet modulus maxima behaved as indicated by (7). In particular, for the original nonscaled case, the ratio of the wavelet modulus maxima for a step discontinuity is given by $M_{2^{2}} f / M_{2^{1}} f=0.75$ and $M_{2^{3}} f / M_{2^{1}} f=0.6875$. The ratio for an impulse is given by $M_{2^{2}} f / M_{2^{1}} f=0.375$ and $M_{2^{2}} f / M_{2^{1}} f=0.171875$. We will exploit this wavelet maxima evolution behavior when determining the deblocking thresholds at different scales.

\section{Deblocking Algorithm}

\section{A. Detection of Block Discontinuities}

The first step in our wavelet deblocking algorithm involves finding appropriate threshold $T_{1}$ for the first wavelet scale. The
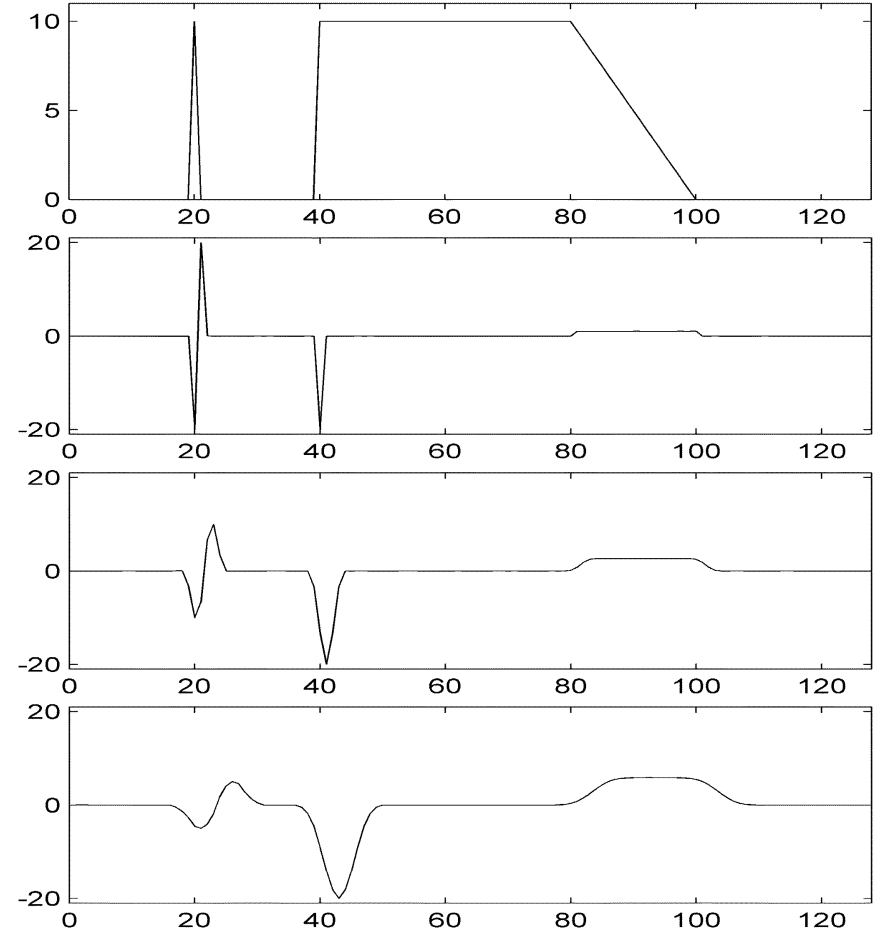

Fig. 2. Top row: a signal with an impulse $(t=20)$, a step discontinuity $(t=$ $40)$, and a ramp ( $t=80$ to $t=100)$. Second row: wavelet transform at scale $2^{1}$. Third row: wavelet transform at scale $2^{2}$. Bottom row: wavelet transform at scale $2^{3}$.

simplest way to compute $T_{1}$ is to let it be equal to the average of all horizontal and vertical block differences in the first wavelet scale. However, some discontinuities at the block boundaries can be due to genuine edges, which happened to be located at the 


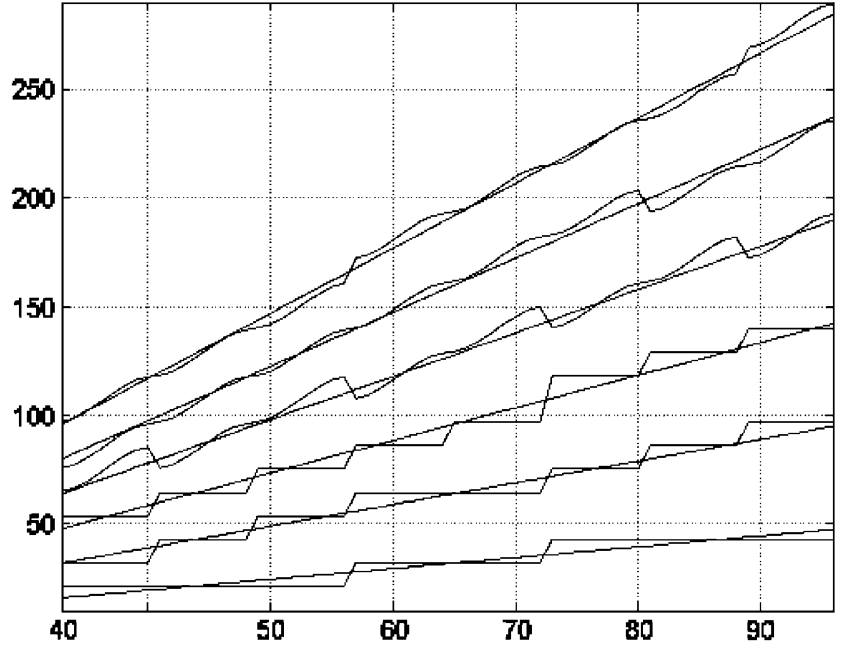

Fig. 3. Horizontal scan lines of six ramp images and the lines of the BDCT compressed images using Q2. The maximum vertical block discontinuity of 21.5 occurs at position $72-73$ on the third plot from the bottom.

$8 \times 8$ block boundaries. Inclusion of these values into the calculation of $T_{1}$ will lead to overestimation since they are much larger than the discontinuities caused by quantization. On the other hand, pixels of a very smooth region at block boundaries may not have block discontinuities caused by quantization. Inclusion of these values will lead to underestimation of $T_{1}$. Thus, the threshold $T_{1}$ should be computed from block boundaries that exhibit block discontinuities due to quantization.

From (6), we see that the block discontinuity due to blocking artifact is constrained by the quantization step size for the dc value. It was also observed that the largest step discontinuity due to quantization is equal to two dc step sizes for a ramp (i.e., constant slope) signal. This observation has been illustrated in Fig. 3, where the horizontal scan lines of six ramp images with different slopes (the slope varies from 0.5 to 3 , in step of 0.5 ) and their BDCT compressed images (using quantization table Q2 in the Appendix) are shown. Using (6), the dc step size for $\mathrm{Q} 2$ is given by $\mathrm{Q} 2(0,0) / 8=86 / 8=10.75$. Fig. 3 shows that the maximum vertical block discontinuities are bounded by twice the dc step size. The maximum block discontinuity is at $\hat{u}(i, 73)-\hat{u}(i, 72)=21.5$, on the third plot, counting from the bottom up.

A smooth region with varying slope produces more complicated BDCT blocking artifacts and may have block discontinuities that are slightly larger than twice the dc step size due to the contribution from high-frequency DCT coefficients. Based on the above consideration, we set the range $R$ where $v b$ or $h b$ is classified as block discontinuity as

$$
R=\left[R_{\min }, R_{\max }\right]=\left[0.5 * \Delta_{d c}, 2.5 * \Delta_{d c}\right]
$$

where $\Delta_{d c}$ is the dc step size associated with the particular quantization table. Given a BDCT compressed image $\hat{f}, R$ can be easily determined since the decoder has knowledge about the particular quantization table used. A block discontinuity map can be computed from $\hat{f}$, where a set pixel in the map signifies the presence of a block discontinuity. Fig. 4 shows the BDCT compressed $512 \times 512$ "Lena" image, and Fig. 5 shows the

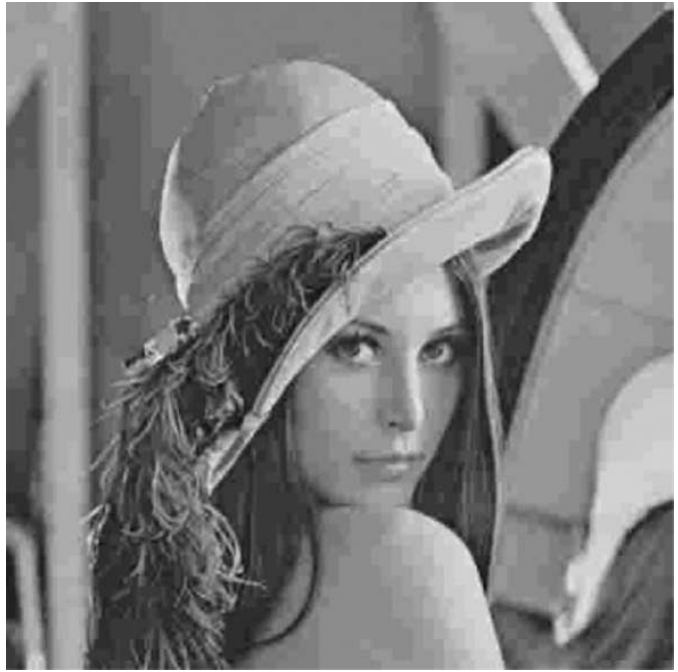

Fig. 4. BDCT compressed $512 \times 512$ "Lena" image using Q2 (30.091 dB).

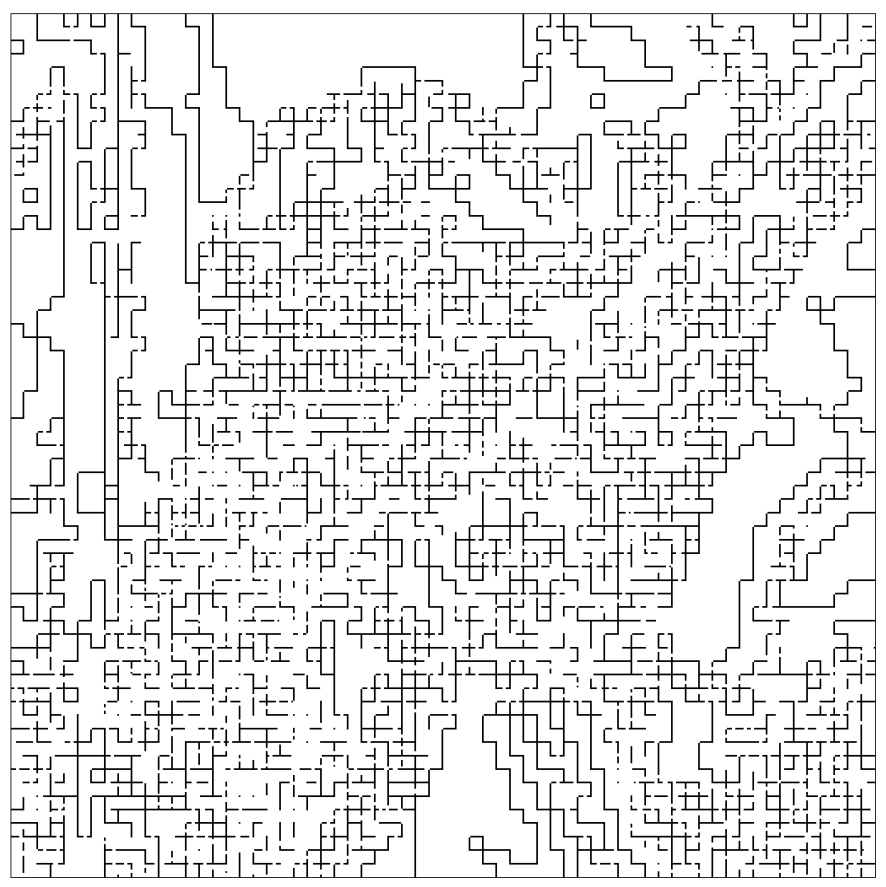

Fig. 5. Block discontinuity map for the BDCT compressed "Lena" of Fig. 4.

corresponding block discontinuity map. The quantization table used is Q2 and $R=[5.375,26.875]$. In computing the block discontinuity map, only horizontal or vertical block discontinuities of length $\geq 4$ are retained. This is in accordance with the observation that block discontinuities due to quantization artifacts are usually line segments and are not isolated points along the block boundary. In addition, $v b$ or $h b$ located at block corners are classified as block discontinuities if the immediate locations to the left and right, or above and below, are block discontinuity, and $|v b|$ or $|h b|$ is smaller than twice $R_{\max }$.

\section{B. Threshold Maps Generation at Different Wavelet Scales}

Our wavelet deblocking algorithm adopts a three-scale wavelet decomposition. Given a BDCT compressed image $\hat{f}$, the three-scale overcomplete wavelet representation of $\hat{f}$ 


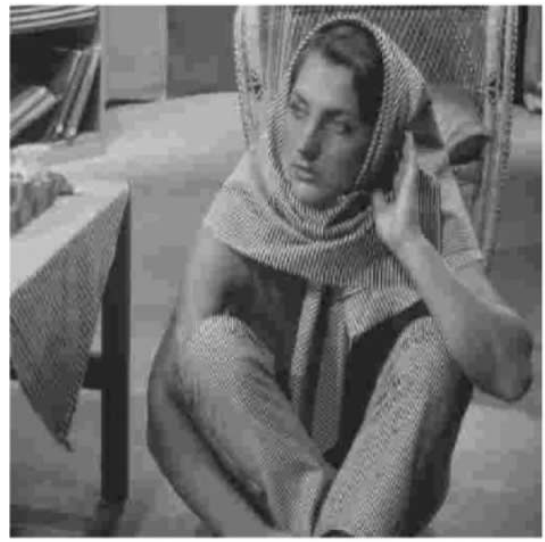

(a)

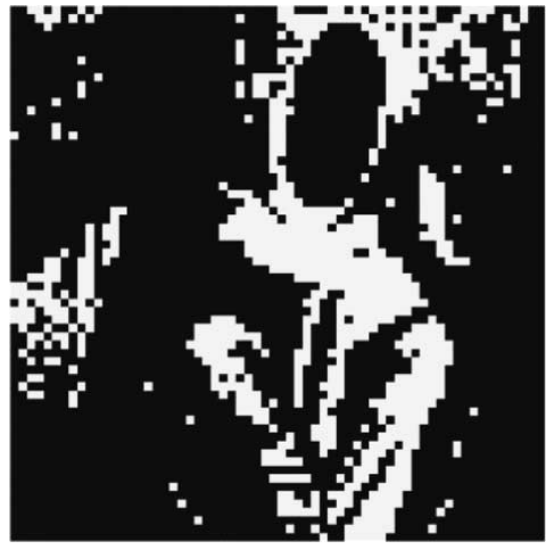

(b)

Fig. 6. (a) The BDCT compressed $512 \times 512$ "Barbara" image using Q2 $(25.591 \mathrm{~dB})$, and (b) the associate texture map.

is computed. Then, the first scale squared-magnitude image $S M_{1}(m, n)$ defined by

$$
S M_{1}(m, n)=\left|W_{2^{1}}^{1} \hat{f}(m, n)\right|^{2}+\left|W_{2^{1}}^{2} \hat{f}(m, n)\right|^{2}
$$

is computed from the first-scale wavelet coefficients $W_{2^{1}}^{1} \hat{f}(m, n)$ and $W_{2^{1}}^{2} \hat{f}(m, n)$. Let $C=\left\{C_{v}, C_{h}\right\}$ be the collection of block discontinuity locations, i.e., where the block discontinuity map is set (see Section IV-A). The subset $C_{v}$ is the collection of vertical block discontinuities, and subset $C_{h}$ is the collection of horizontal block discontinuities. Let $N(C)$ denote the number of elements in $C$. The threshold $T_{1}$ is computed by

$$
T_{1}=\frac{\left[\sum_{(m, n) \in C_{v}} S M_{1}(m, n)+\sum_{(m, n) \in C_{h}} S M_{1}(m, n)\right]}{N(C)} .
$$

Recall from Section III that the ratio of the wavelet modulus maxima for a step discontinuity is given by $M_{2^{2}} f / M_{2^{1}} f=$ 0.75 and $M_{2^{3}} f / M_{2^{1}} f=0.6875$, and the ratio for an impulse is given by $M_{2^{2}} f / M_{2^{1}} f=0.375$ and $M_{2^{2}} f / M_{2^{1}} f=0.171875$. In our wavelet deblocking algorithm, the ratio $\sqrt{T_{2} / T_{1}}$ is taken to be the average of wavelet modulus maxima responses for a step discontinuity and an impulse, i.e., $\sqrt{T_{2} / T_{1}}=(0.75+$ $0.375) / 2=0.5625$. Similarly, we set $\sqrt{T_{3} T_{1}}=(0.6875+$ $0.171875) / 2=0.4297$. Thus, the threshold $T_{2}$ at scale two and threshold $T_{3}$ at scale three are given by

$$
\begin{aligned}
& T_{2}=0.3164 T_{1} \\
& T_{3}=0.1846 T_{1} .
\end{aligned}
$$

From the evolution behavior of wavelet modulus maxima across scales, one can observe that discontinuities and impulse-like structures in an image have a strong showing in the first wavelet scale and decay rapidly as one goes up in scale.
Hence, a simple lowpass filtering along the block boundaries can effectively suppress these artifacts. Specifically, we replace those pixels along the vertical and horizontal block boundaries as follows:

$$
\begin{aligned}
& W_{2^{1}}^{1} \hat{f}(m, 8 n) \\
& \quad \leftarrow \frac{\left(W_{2^{1}}^{1} \hat{f}(m, 8 n-1)+W_{2^{1}}^{1} \hat{f}(m, 8 n)+W_{2^{1}}^{1} \hat{f}(m, 8 n+1)\right)}{3} \\
& \quad m=0, \ldots, M-1 ; \quad n=1, \ldots, \frac{N}{8}-1 \\
& W_{2^{1}}^{2} \hat{f}(m, 8 n) \\
& \quad \leftarrow \frac{\left(W_{2^{1}}^{2} \hat{f}(8 m-1, n)+W_{2^{1}}^{2} \hat{f}(8 m, n)+W_{2^{1}}^{2} \hat{f}(8 m+1, n)\right)}{3} \\
& \quad m=1, \ldots, \frac{M}{8}-1 ; \quad n=0, \ldots, N-1 .
\end{aligned}
$$

Given the thresholds $T_{1}, T_{2}$, and $T_{3}$, the next step in our deblocking algorithm is the computation of threshold maps at different wavelet scales. Since the texture pattern has rapid variations in intensity, the wavelet representation has an impulse-like behavior. In particular, most of the energy of the texture is concentrated in the first wavelet scale. To prevent oversmoothing of the texture region, the first scale threshold is set to be equal to $T_{1}$ for a nontexture region and $0.8 T_{1}$ for a texture region. An 8 $\times 8$ block is classified as a texture block if the block contains significant high-frequency coefficients [9]. Specifically, if any of the DCT coefficient $\hat{v}_{a, b}(i, j)$, for $i+j>3$, is nonzero, then the block indexed by $(a, b)$ is a texture block. Fig. 6(b) shows the texture map for the BDCT compressed image "Barbara" using Q2 shown in Fig. 6(a), where texture pattern can be found on her clothing, the tablecloth, and the rattan chair.

With the texture region defined, and given the squared-magnitude map $S M_{1}$ as defined by (9), the threshold map $t m a p_{1}$ is computed as

$$
\operatorname{tmap}_{1}(m, n)=\left\{\begin{array}{cc}
1, & \text { if } S M_{1}(m, n)>T_{1} \\
& \text { for nontexture region } \\
1, & \text { if } S M_{1}(m, n)>0.8 T_{1} \\
& \text { for texture region } \\
0, & \text { otherwise. }
\end{array}\right.
$$




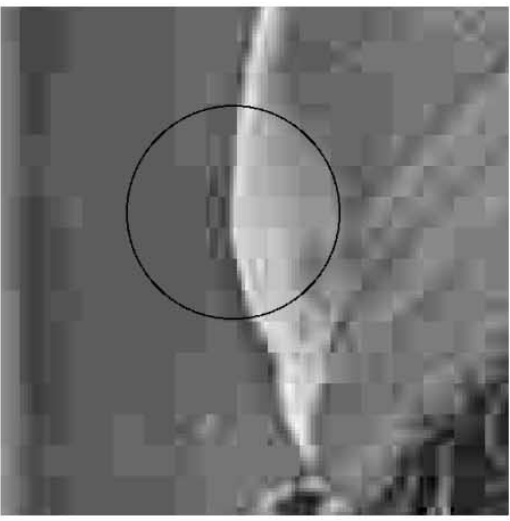

(a)

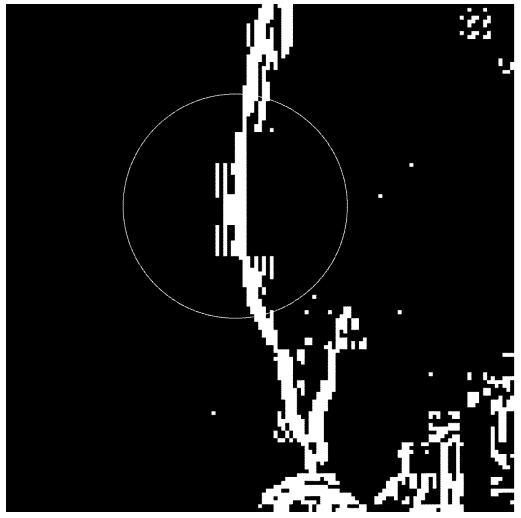

(b)

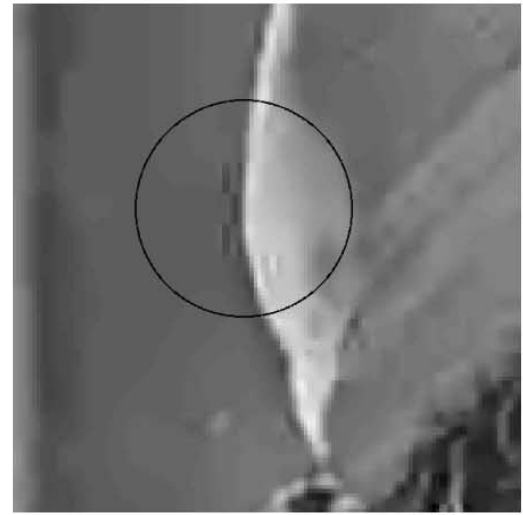

(c)

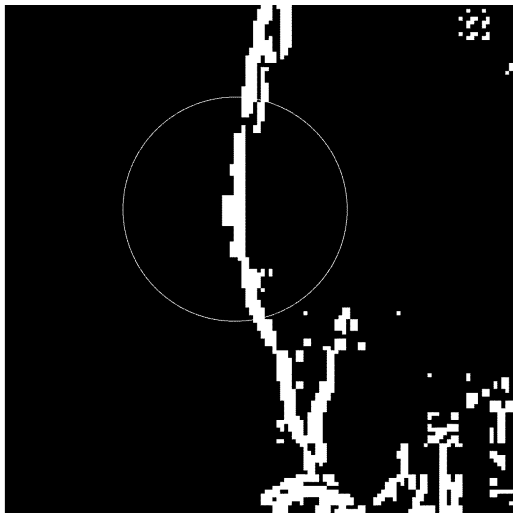

(d)

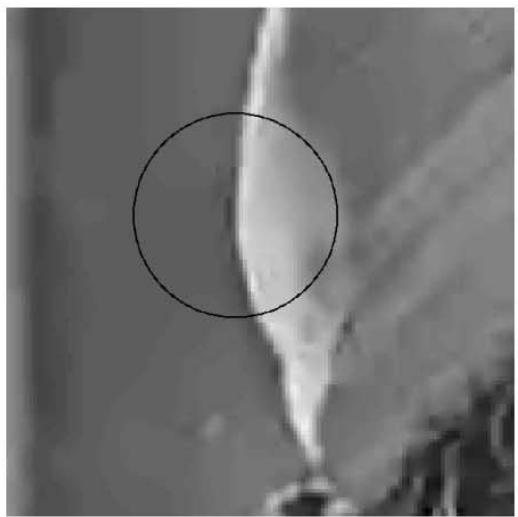

(e)

Fig. 7. (a) A portion of the BDCT compressed "Lena" image using Q2 showing ringing artifact, (b) tmap 1 before deringing, (c) deblocked image without deringing, (d) $t m a p_{1}$ after deringing, and (e) deblocked image with deringing applied.

The threshold maps for scales 2 and 3, tmap $_{2}$ and $\operatorname{tmap}_{3}$, are given by

$$
\operatorname{tmap}_{k}(m, n)=\left\{\begin{array}{ll}
1, & \text { if } S M_{k}(m, n)>T_{k} \\
0, & \text { otherwise }
\end{array} \quad k=2,3 .\right.
$$

In computing the overcomplete wavelet representation, the BDCT compressed image is assumed to be periodically extended in both directions. Hence, discontinuities in gray level along the image boundary will show up in the wavelet representation. However, these discontinuities are not artifacts of the BDCT compression and should not be suppressed. The extent of support of the wavelet kernels at different scales indicated that we should set the following rows and columns of tmap $_{k}$ to 1 . They are the first row and first column of $t m a p_{1}$, the first four rows and four columns, as well as the last row and last column of $\mathrm{tmap}_{2}$, and the first ten rows and ten columns, as well as the last three rows and three columns of tmap $_{3}$.

\section{Ringing Artifact Suppression at First Wavelet Scale}

Besides the block discontinuities, coarse quantization would also produce ringing near strong edges due to the truncation of high-frequency DCT coefficients [9]-[11]. This artifact is visually very annoying even though it does not usually cause much degradation in terms of peak SNR (PSNR) value. Since strong edges could locate well inside an $8 \times 8$ block, smoothing along block boundaries does not suppress this kind of artifact. A better strategy is to locate such strong edges, whether they are on a block boundary or inside a block, and then smooth out the ringing while preserving the sharpness of the edges. As ringing artifact exhibits impulse-like behavior in the wavelet domain, i.e., it has a strong showing at the first wavelet scale and decays rapidly at higher scales; it can be effectively suppressed by forcing the corresponding first-scale wavelet coefficients to zero. The ringing artifact produces a band-like pattern of $\{0,1,0,1,0, \ldots\}$ near strong edges in $\operatorname{tmap}_{1}$ due to the response of a two-tap high-pass wavelet filter to ringing feature. By removing this band-like pattern in $t m a p_{1}$, thereby zeroing out the first-scale wavelet coefficients at those positions, ringing artifact can be suppressed. The strong vertical and horizontal edges in the image are found by applying the Sobel edge operator to the BDCT compressed image. Then, the $\operatorname{tmap}_{1}$ can be searched horizontally and vertically near the vicinity of strong edges to detect such band-like pattern. The search is through an exact pattern matching, i.e., the detection of a group of consecutive $\{010\}$ pixels. Finally, the set pixels in $\operatorname{tmap}_{1}$ that correspond to such a pattern are set to zero, thus effectively removing the band-like pattern.

After computing the threshold maps at scales $j=1,2$, and 3 , the modified wavelet coefficients at scales 1,2 , and 3 are obtained by

$$
W_{2^{j}}^{k} \hat{f}(m, n)= \begin{cases}W_{2^{j}}^{k} \hat{f}(m, n), & \text { if } \operatorname{tmap}_{j}(m, n)=1 \\ 0, & \text { otherwise }\end{cases}
$$

where $j=1,2,3, k=1,2, m=0,1, \ldots, M-1$, and $n=0,1, \ldots, N-1$. Finally, the wavelet deblocked image $\tilde{f}$ 


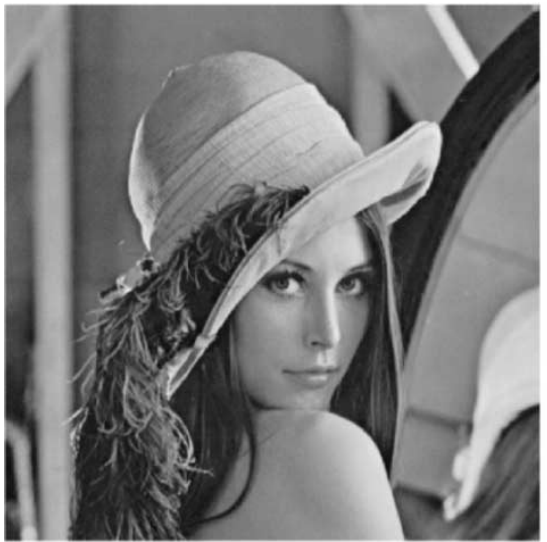

(a)

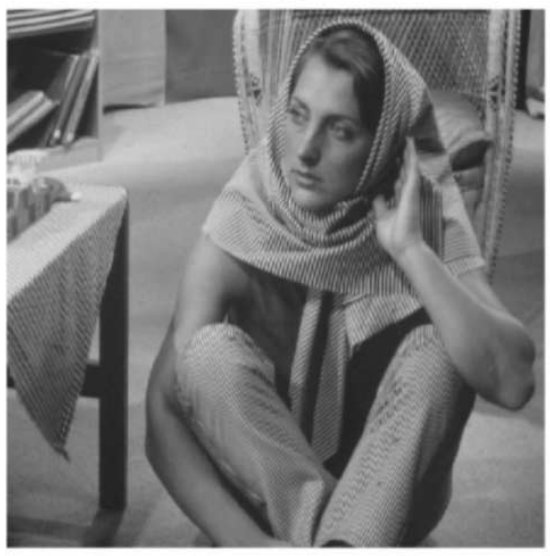

(c)

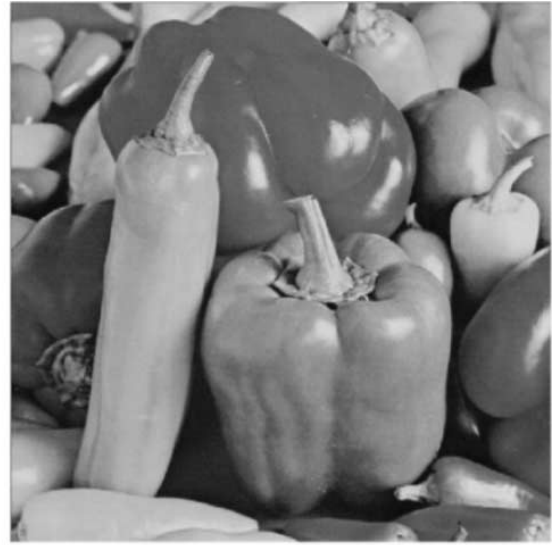

(b)

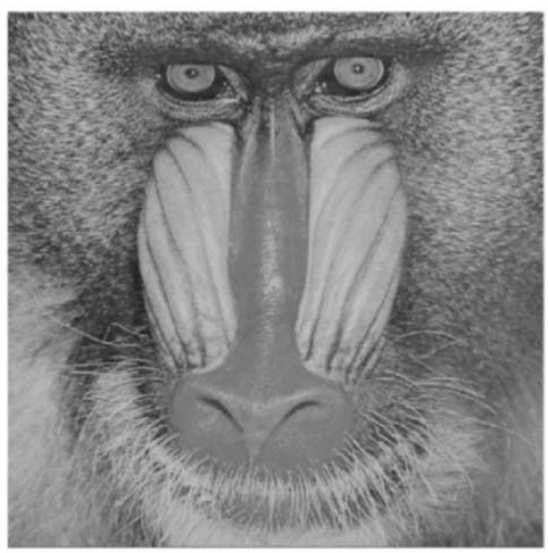

(d)

Fig. 8. Four original images of size 512 by 512: (a) “Lena," (b) "Peppers,” (c) "Barbara," and (d) "Baboon."

is obtained from the modified overcomplete wavelet representation by an inverse wavelet transform using (3). In our algorithm, the coarse image $S_{2^{3}} \hat{f}$ is not modified since the blocking artifacts have a negligible effect on the coarse image at scale 3 .

To illustrate the effectiveness of our deringing procedure, we show in Fig. 7 a portion of the "Lena" image around the hat, where severe ringing can be observed along the edge of the hat. Fig. 7(a) is the BDCT compressed image using quantization table Q2. Fig. 7(b) is the $\operatorname{tmap}_{1}$ before deringing. A band-like pattern can be observed where ringing occurs. Fig. 7(c) is the deblocked image obtained using our algorithm, but without deringing. Clearly, the ringing artifact is still present in the deblocked image. Fig. 7(d) is the $\operatorname{tmap}_{1}$ after deringing; the band-like pattern has been eliminated. Fig. 7(e) is the deblocked image obtained with deringing applied. The ringing artifact has been effectively suppressed.

\section{Enforcement of DCT Quantization Constraint and Range Constraint}

In BDCT compression, a quantization table is used to quantize the continuous-valued DCT coefficients into discrete values. The quantization constraint formulated from the quantization table restricts the value of the DCT coefficients to be bounded by the quantization interval. It is obvious that the original image as well as the BDCT compressed image satisfy the quantization constraint. The quantization constraint set has been widely used to constraint the final deblocked image in POCS-based deblocking algorithms [3]-[6], [11], [24]. In principle, any deblocked image that is consistent with the original image should satisfy the quantization constraint.

The image $\tilde{f}$ usually does not satisfy the DCT quantization constraint. By projecting $\widetilde{f}$ onto the quantization constraint set, one can obtain a deblocked image consistent with the original image. Let $\widetilde{v}$ be the set of DCT coefficients of $\widetilde{f}$. The projection is achieved by modifying the $(i, j)$ th DCT coefficient at block $(a, b)$, for all $i, j=0, \ldots, 7, a=0, \ldots, M / 8-1$, $b=0, \ldots, N / 8-1$, as follows:

$$
\widetilde{v}_{a, b}(i, j)= \begin{cases}F_{\max }(i, j), & \text { if } \widetilde{v}_{a, b}(i, j)>F_{\max }(i, j) \\ F_{\min }(i, j), & \text { if } \widetilde{v}_{a, b}(i, j)<F_{\min }(i, j) \\ \widetilde{v}_{a, b}(i, j), & \text { otherwise }\end{cases}
$$

where

$$
\begin{aligned}
F_{\max }(i, j) & =\left(q_{a, b}(i, j)+0.5\right) \Delta(i, j) \\
F_{\min }(i, j) & =\left(q_{a, b}(i, j)-0.5\right) \Delta(i, j) \\
q_{a, b}(i, j) & =\text { round to nearest integer }\left[\frac{v_{a, b}(i, j)}{\Delta(i, j)}\right]
\end{aligned}
$$

and $v$ is the DCT coefficients of the original image and $\Delta(i, j)$ is the quantization interval of the $(i, j)$ th DCT coefficient. Note that it is the set of $q$ (not $v$ ) that is available at the decoder. The deblocked image is then obtained by performing an inverse BDCT on the modified coefficients.

Although the deblocked image obtained after projection onto the quantization constraint set of (16) and (17) is consistent 


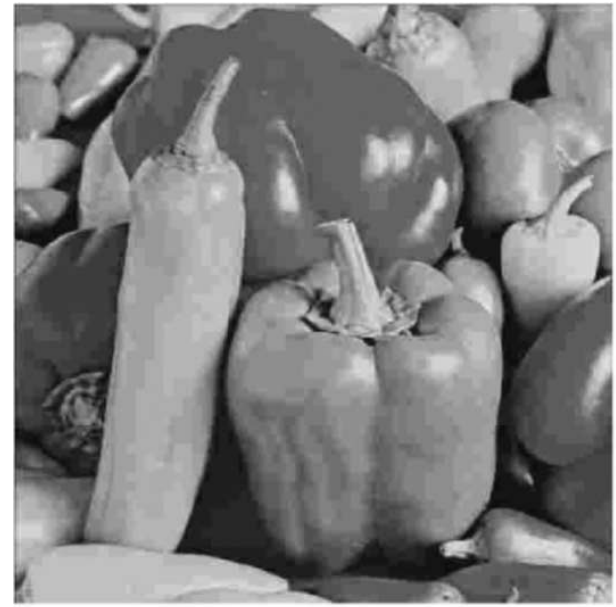

(a)

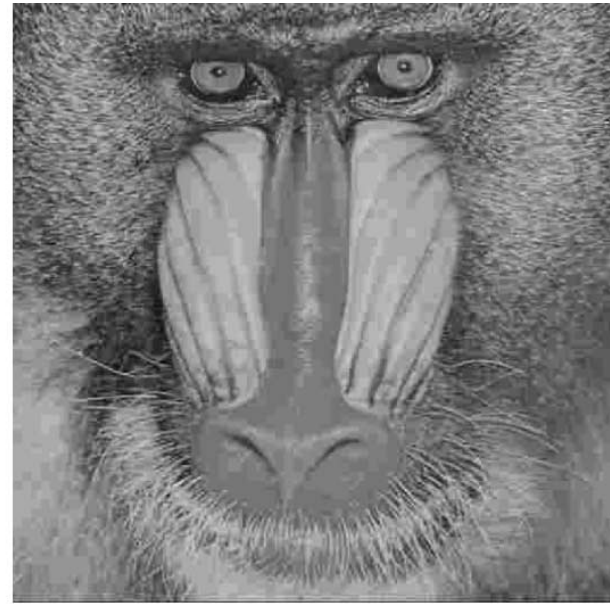

(b)

Fig. 9. BDCT compressed images using Q2. The PSNRs are: (a) $29.808 \mathrm{~dB}$ for "Peppers" and (b) $24.143 \mathrm{~dB}$ for "Baboon."

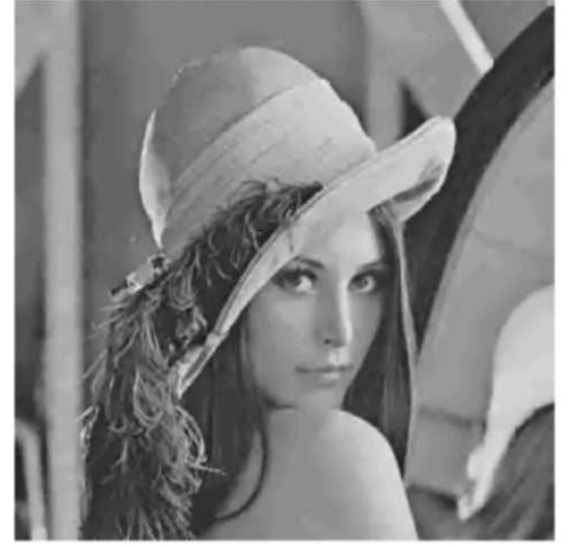

(a)

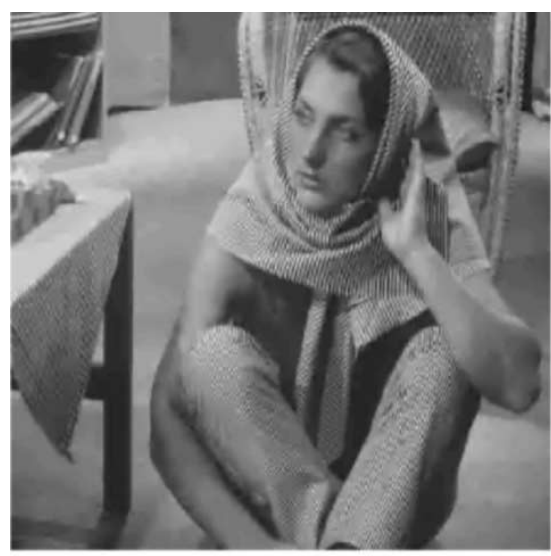

(c)

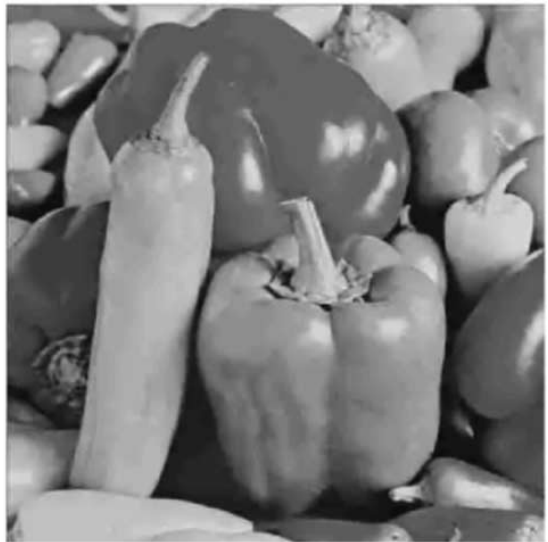

(b)

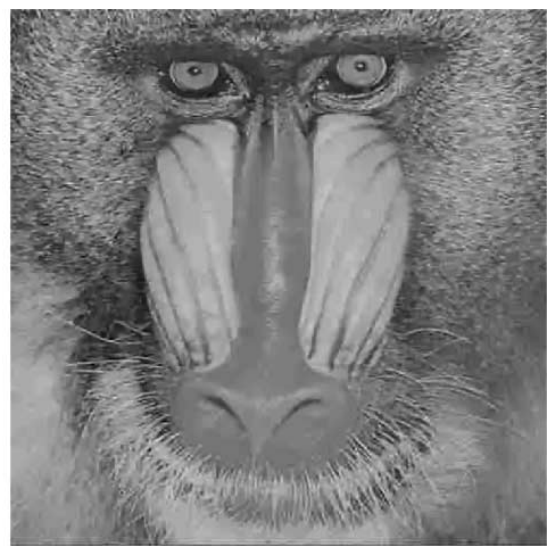

(d)

Fig. 10. Deblocked images using the proposed algorithm for Q2. Blocking artifacts are suppressed while image details are well preserved. The PSNRs are: (a) $31.187 \mathrm{~dB}$, (b) $30.966 \mathrm{~dB}$, (c) $26.043 \mathrm{~dB}$, and (d) $24.450 \mathrm{~dB}$, respectively.

with the original image, Park and Kim [24] have shown that projection onto the narrow quantization constraint set (NQCS) can produce better results in term of PSNR. Instead of (17), the NQCS has $F_{\min }$ and $F_{\max }$ given by

$$
\begin{aligned}
F_{\max }(i, j) & =\left(q_{a, b}(i, j)+\alpha\right) \Delta(i, j) \\
F_{\min }(i, j) & =\left(q_{a, b}(i, j)-\beta\right) \Delta(i, j)
\end{aligned}
$$

with $0<\alpha, \beta \leq 0.5$. Our experience has indicated that a value of $\alpha=\beta=0.3$ generally gives good results.

The last step in our deblocking algorithm is the application of a range constraint onto the deblocked image. Since the original image is represented by $8 \mathrm{~b}$ and has a range of [0-255], we require that the deblocked image also stays within this range. This is done by projecting any image value outside of the range [0-255] into this range. 
TABLE I

PSNR Performance (IN dB) of Different Deblocking Algorithms in COMPaRison With our Algorithm. The Best PSNR IN EACH COLUMN IS Shown in Bold Font. The Proposed Algorithm Ranks Well With the Other Deblocking Algorithms

Q1

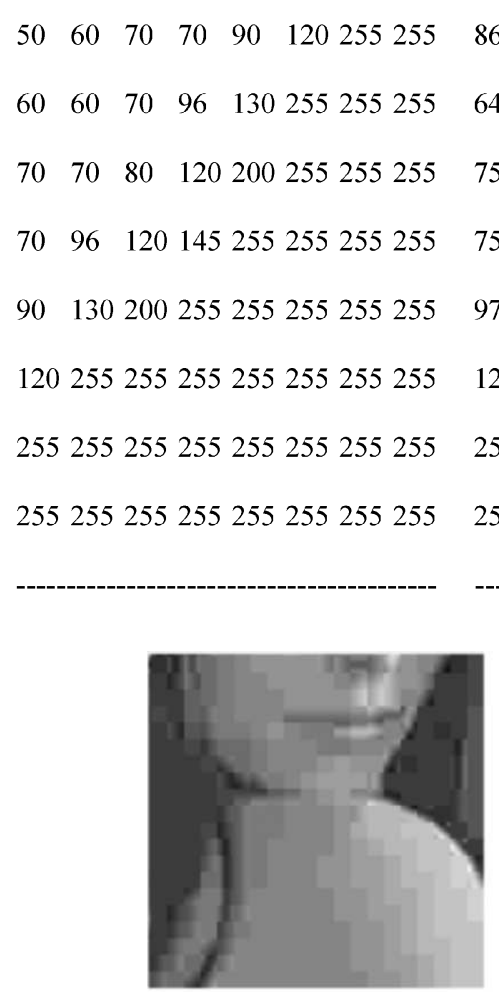

(a)

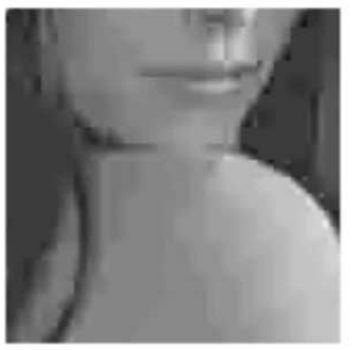

(d)

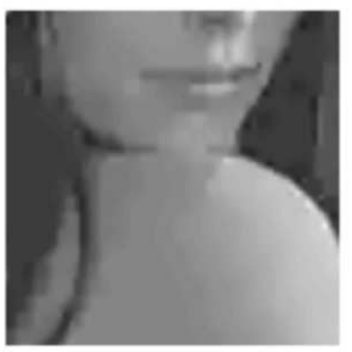

(g)
Q2

$\begin{array}{llllllll}86 & 59 & 54 & 86 & 129 & 216 & 255 & 255\end{array}$

$\begin{array}{llllllll}64 & 64 & 75 & 102 & 140 & 255 & 255 & 255\end{array}$

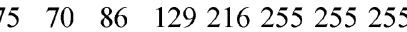

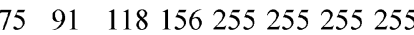

$97 \quad 118199255255255255255$

129189255255255255255255

255255255255255255255255

255255255255255255255255
Q3

130150192255255255255255

150192255255255255255255

192255255255255255255255

255255255255255255255255

255255255255255255255255

255255255255255255255255

255255255255255255255255

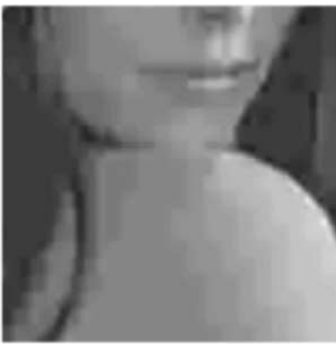

(b)

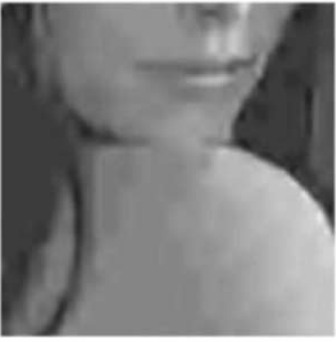

(e)

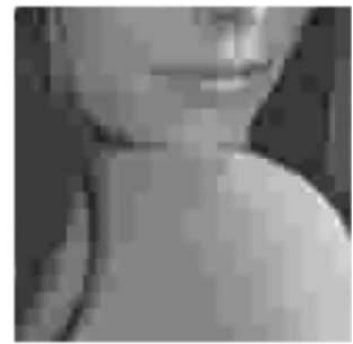

(c)

(f)

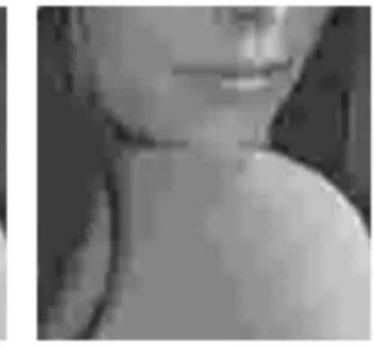

Fig. 11. Deblocked results around the shoulder of "Lena." (a) BDCT compressed with Q3, (b) Xiong's algorithm, (c) Hsung's algorithm, (d) MPEG4's algorithm, (e) Paek's algorithm, (f) Yang's algorithm, and (g) our algorithm.

\section{EXPERIMENTAL RESULTS}

To illustrate the performance of our algorithm, we have carried out extensive deblocking experiments using images with different characteristics. The deblocking results on four
512 × 512 images, i.e., "Lena," "Peppers," "Barbara," and "Baboon" shown in Fig. 8, using the three quantization tables in the Appendix, are presented here. "Lena" and "Peppers" contain mainly smooth regions. Hence, any lowpass smoothing type of deblocking algorithm will be effective on these images. 


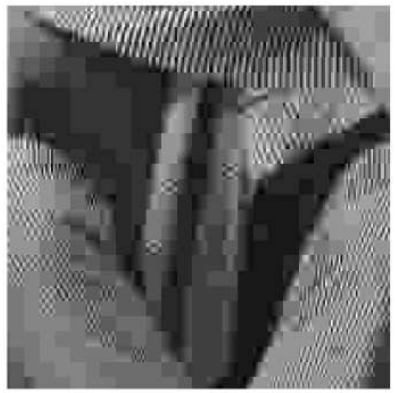

(a)

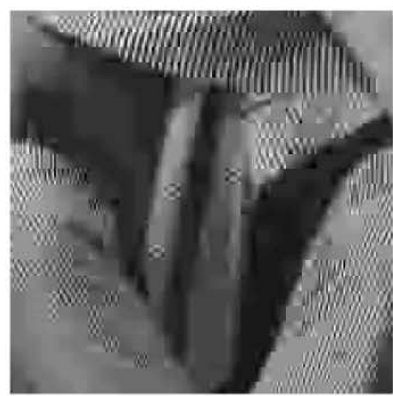

(d)

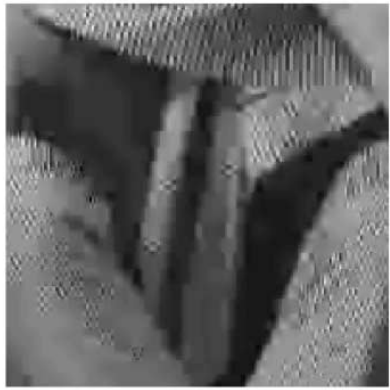

(b)

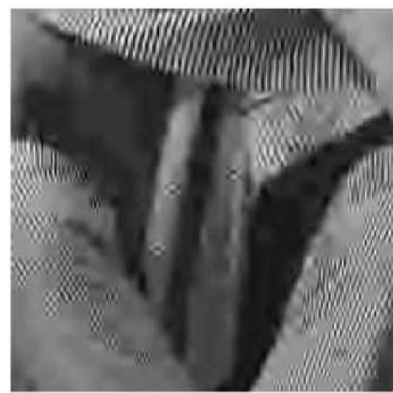

(e)

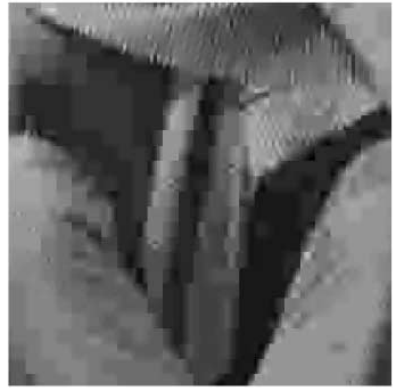

(c)

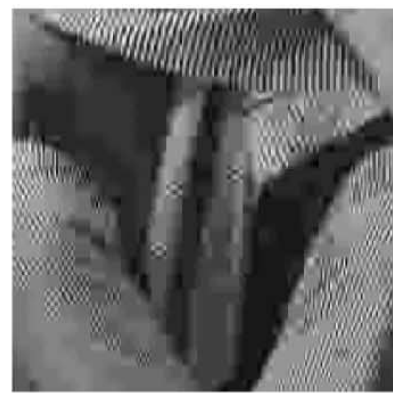

(f)

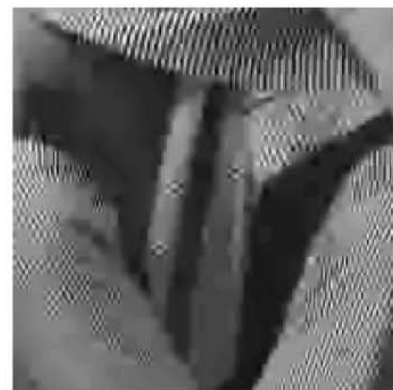

(g)

Fig. 12. Deblocked results around the leg of "Barbara.” (a) BDCT compressed with Q3, (b) Xiong's algorithm, (c) Hsung's algorithm, (d) MPEG4's algorithm, (e) Paek's algorithm, (f) Yang's algorithm, and (g) our algorithm.

"Lena" also contains a texture-like hair region. Since humans are attentive to facial and skin regions, the head-and-shoulder "Lena" image is useful for evaluating the detailed visual performance of the algorithm. The "Barbara" image contains a regular texture pattern on her garment, the tablecloth, and the rattan chair at the back whereas the "Baboon" image contains a large area of hair region, which has a random-like texture characteristic. These four images should serve to illustrate the effectiveness and the robustness of our deblocking algorithm in handling different image content.

The four BDCT compressed images using Q2 are shown in Figs. 4, 6(a), and 9(a) and (b). Blocking artifacts can be readily observed on all four images. Fig. 10 shows the corresponding deblocked images obtained from our algorithm. The blocking artifacts have been effectively suppressed while image details are well preserved.

A comparative study has been done with a few of the popular deblocking algorithms in the literature as well as the postfiltering algorithm of the MPEG4-Video Verification Model. In particular, the quantitative (in terms of PSNR) as well as the visual performance of our algorithm are compared with Xiong's wavelet deblocking $\left(\mathrm{WD}_{\mathrm{x}}\right)$ algorithm [21], Hsung's wavelet singularity detection $\left(\mathrm{WSD}_{\mathrm{H}}\right)$ algorithm [23], the MPEG4-VM postfiltering (MPG4) algorithm [10], Paek's POCS (POCS $\mathrm{P}$ ) algorithm [6], and Yang's spatially adaptive POCS $\left(\mathrm{POCS}_{\mathrm{y}}\right)$ algorithm [5]. The former three are noniterative deblocking methods while the latter two are iterative deblocking methods. In Yang's algorithm, the smoothness constraint is also applied to one off-boundary row/column, both to the left and to the right of the block boundaries. For the two iterative POCS algorithms, we set the number of iterations to five. Since POCS does not guarantee convergence to the original image but only to an image in the intersection of the constraint sets, the PSNR at the fifth iteration is not necessarily the maximum PSNR. For example, the best PSNR for "Lena" is achieved at the third iteration for Paek's algorithm. The PSNR results for the different algorithms are tabulated in Table I. It can be seen that our algorithm consistently outperformed the three noniterative algorithms in terms of PSNR. Our algorithm also compared favorably to the two iterative POCS algorithms.

For visual evaluation, we show in Fig. 11 the deblocked results around the shoulder of "Lena" obtained from different algorithms. The BDCT compression is done with Q3. Visual inspection clearly indicated that our algorithm produces the most visually pleasing result. In Fig. 12, we show the deblocked results around the leg of "Barbara," which consists mainly of texture region. Visual inspection indicated that our algorithm is effective at removing block discontinuities in a smooth region while preserving the sharpness of the texture region. Finally, in terms of computational cost, MPEG4 postfiltering is the lowest, whereas our algorithm is comparable in speed to Xiong's wavelet deblocking algorithm.

\section{CONCLUSION}

Based on a theoretical analysis of blocking artifacts, we proposed a simple yet effective wavelet-based deblocking algorithm using the three-scale overcomplete wavelet scheme. We showed that block discontinuities along block boundaries are constrained by the de quantization interval of the quantization table and use this fact to locate image pixels that correspond to block discontinuities. The threshold for the first wavelet scale can then be computed based on the wavelet coefficients at these locations. The thresholds for the subsequent two scales are obtained based on the behavior of wavelet modulus maxima evolution across scales for step discontinuities and impulse-like sin- 
TABLE II

QUANTIZATION TABLES FOR BCT

\begin{tabular}{c|c|c|c|c|c|c|c|c|c|c|c|c}
\hline & \multicolumn{3}{|c|}{ LENNA } & \multicolumn{3}{c|}{ PEPPERS } & \multicolumn{3}{c}{ BARBARA } & \multicolumn{3}{c}{ BABOON } \\
\hline Quantizer & Q1 & Q2 & Q3 & Q1 & Q2 & Q3 & Q1 & Q2 & Q3 & Q1 & Q2 & Q3 \\
\hline Test image & 30.702 & 30.091 & 27.382 & 30.413 & 29.808 & 27.218 & 25.939 & 25.591 & 24.028 & 24.320 & 24.143 & 22.133 \\
\hline WD $_{\mathrm{x}}[21]$ & 31.215 & 30.758 & 28.315 & 30.650 & 30.224 & 28.173 & 25.226 & 25.070 & 24.100 & 24.240 & 24.125 & 22.476 \\
\hline WSD $_{\mathrm{H}}[23]$ & 31.299 & 30.696 & 27.894 & 31.149 & 30.563 & 27.856 & 24.652 & 24.544 & 23.631 & 24.307 & 24.160 & 22.391 \\
\hline MPG4 [10] $^{3}$ & 31.211 & 30.694 & 28.095 & 31.180 & 30.687 & 28.256 & 26.092 & 25.774 & 24.367 & 24.451 & 24.293 & 22.401 \\
\hline POCS $_{\mathrm{P}}[6]$ & $\mathbf{3 1 . 6 2 9}$ & 31.020 & 28.513 & 31.199 & 30.642 & 28.344 & $\mathbf{2 6 . 6 3 8}$ & $\mathbf{2 6 . 3 1 6}$ & $\mathbf{2 4 . 7 3 3}$ & $\mathbf{2 4 . 6 3 1}$ & $\mathbf{2 4 . 4 6 9}$ & 22.522 \\
\hline POCS & & & & & & & & & & & & \\
\hline Our method & 31.314 & 30.741 & 28.294 & 30.947 & 30.403 & 28.098 & 26.399 & 26.051 & 24.449 & 24.545 & 24.386 & 22.414 \\
\hline
\end{tabular}

gular image structures. Since ringing artifacts behave as impulse-like structure in the wavelet domain, they are effectively suppressed by thresholding the appropriate first scale wavelet coefficients, which are located by searching for a specific pattern in accordance to the filter response of the highpass wavelet filter, to zero. After processing the wavelet coefficients, the deblocked image is obtained by an inverse wavelet transform. To ensure that the deblocked image is consistent with the BDCT compressed image, the quantization constraint and the range constraint are also applied onto the deblocked image. The proposed algorithm can suppress both block discontinuities and ringing artifacts effectively while preserving true edges and textural information. Comparative study using images of different characteristics has shown that our algorithm consistently outperform several iterative and noniterative algorithms in both PSNR and visual quality.

\section{APPENDIX}

The quantization tables for BCT are shown in Table II.

\section{REFERENCES}

[1] N. Ahmad, T. Natarajan, and K. R. Rao, "Discrete cosine transform coding of images," IEEE Trans. Comput., vol. C-23, pp. 90-93, 1941.

[2] H. C. Reeves and J. S. Lim, "Reduction of blocking effects in image coding," J. Opt. Eng., vol. 23, pp. 34-37, 1984.

[3] A. Zakhor, "Iterative procedures for reduction of blocking effects in transform image coding," IEEE Trans. Circuits Syst. Video Technol., vol. 2, pp. 91-95, Mar. 1992.

[4] Y. Yang, N. P. Galatsanos, and A. K. Katsaggelos, "Regularized reconstruction to reduce blocking artifacts of block discrete cosine transform compressed images," IEEE Trans. Circuits Syst. Video Technol., vol. 3, pp. 421-432, Dec. 1993.

[5] Y. Yang and N. P. Galatsanos, "Projection-based spatially adaptive reconstruction of block-transform compressed images," IEEE Trans. Image Processing, vol. 4, pp. 896-908, July 1995.

[6] H. Paek, R. C. Kim, and S. U. Lee, "On the POCS-based postprocessing techniques to reduce the blocking artifacts in transform coded images," IEEE Trans. Circuits Syst. Video Technol., vol. 8, pp. 358-367, June 1998.

[7] S. D. Kim, J. Yi, H. M. Kim, and J. B. Ra, "A deblocking filter with two separate mode in block-based video coding," IEEE Trans. Circuits Syst. Video Technol., vol. 9, pp. 156-160, Feb. 1999.

[8] J. Chou, M. Crouse, and K. Ramchandran, "A simple algorithm For removing blocking artifacts in block-transformed coded images," in Proc. ICIP'98, vol. 1, Oct. 1998, pp. 377-380.
[9] H. W. Park and Y. L. Lee, "A postprocessing method for reducing quantization effects in low bit-rate moving picture coding," IEEE Trans. Circuits Syst. Video Technol., vol. 9, pp. 161-171, Feb. 1999.

[10] MPEG 4 Verification Model, VM 14.2, pp. 260-264, 1999.

[11] Y. Yang and N. P. Galatsanos, "Removal of compression artifacts using projections onto convex sets and line process modeling," IEEE Trans. Image Processing, vol. 6, pp. 1345-1357, Oct. 1997.

[12] S. W. Hong, Y. H. Chan, and W. C. Siu, "A new approach for real-time reduction of blocking effect," Signal Processing, vol. 65, no. 3, pp. 337-346, Mar. 1998

[13] D. L. Donoho, "Denoising by soft-thresholding," IEEE Trans. Inform. Theory, vol. 41, pp. 613-627, May 1995.

[14] D. L. Donoho and M. Johnstone, "Ideal spatial adaptation via wavelet shrinkage," Biometrika, vol. 81, pp. 425-455, 1994.

[15] R. A. Gopinath, M. Lang, H. Guo, and J. E. Odegard, "Wavelet-based post-processing of low bit rate transform coded images," in Proc. ICIP'94, Nov. 1994, pp. 913-917.

[16] D. Wei and C. S. Burrus, "Optimal wavelet thresholding for various coding schemes," in Proc. ICIP'95, Oct. 1995, pp. 610-613.

[17] S. Mallat and S. Zhong, "Characterization of signals from multiscale edges," IEEE Trans. Pattern Anal. Machine Intell., vol. 14, pp. 710-732, July 1992.

[18] S. Mallat and W. L. Hwang, "Singularity detection and processing with wavelets," IEEE Trans. Inform. Theory, vol. 38, pp. 617-643, Mar. 1992.

[19] A. W. C. Liew and N. F. Law, "Reconstruction from 2-D wavelet transform modulus maxima using projection," IEE Proc.-Vision, Image and Signal Processing, vol. 147, no. 2, pp. 176-184, Apr. 2000.

[20] T. C. Hsung, D. P. K. Lun, and W. C. Siu, "A deblocking technique for block-transform compressed image using wavelet transform modulus maxima," IEEE Trans. Image Processing, vol. 7, pp. 1488-1496, Oct. 1998.

[21] Z. Xiong, M. T. Orchard, and Y. Zhang, "A deblocking algorithm for JPEG compressed images using overcomplete wavelet representation," IEEE Trans. Circuits Syst. Video Technol., vol. 7, pp. 433-437, Apr. 1997.

[22] N. C. Kim, I. H. Jang, D. H. Kim, and W. H. Hong, "Reduction of blocking artifact in block-coded images using wavelet transform," IEEE Trans. Circuits Syst. Video Technol., vol. 8, pp. 253-257, June 1998.

[23] T. C. Hsung and D. P. K. Lun, "Application of singularity detection for the deblocking of JPEG decoded images," IEEE Trans. Circuits Syst. II, vol. 45, pp. 640-644, May 1998.

[24] S. H. Park and D. S. Kim, "Theory of projection onto the narrow quantization constraint set and its application," IEEE Trans. Image Processing, vol. 8, pp. 1361-1373, Oct. 1999.

[25] N. F. Law and W. C. Siu, "Successive structural analysis using wavelet transform for blocking artifacts suppression," Signal Processing, vol. 81, no. 7, pp. 1373-1387, July 2001

[26] C. Weerasinghe, A. W. C. Liew, and H. Yan, "Artifact reduction in compressed images based on region homogeneity constraints using the projection onto convex sets algorithm," IEEE Trans. Circuits Syst. Video Technol., vol. 12, pp. 891-897, Oct. 2002.

[27] S. Wu, H. Yan, and Z. Tan, "An efficient wavelet based de-blocking algorithm for highly compressed images," IEEE Trans. Circuits Syst. Video Technol., vol. 11, pp. 1193-1198, Nov. 2001. 


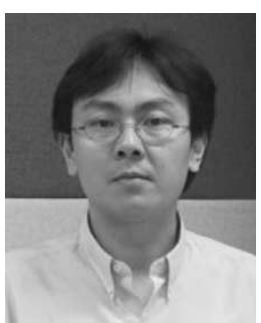

and bioinformatics.
Alan W.-C. Liew (M'03) received the B.E. degree (with first class honors) in electrical and electronic engineering from the University of Auckland, Auckland, New Zealand, in 1993 and the Ph.D. in electronic engineering from the University of Tasmania, Australia, in 1997.

$\mathrm{He}$ is currently a Senior Research Fellow with the Department of Computer Engineering and Information Technology, City University of Hong Kong. His current research interests include wavelets, signal and image processing, pattern recognition,

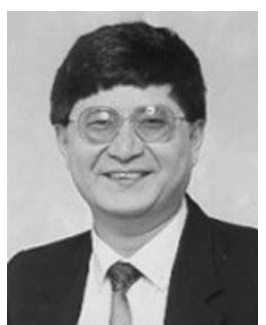

Hong Yan (S'88-M'89-SM'93) received a B.E. degree from Nanking Institute of Posts and Telecommunications, Nanking, China, in 1982, the M.S.E. degree from the University of Michigan, Ann Arbor, in 1984, and the Ph.D. degree from Yale University, New Haven, CT, in 1989, all in electrical engineering.

In 1982 and 1983, he worked on signal detection and estimation as a graduate student and Research Assistant at Tsinghua University, Beijing, China. From 1986 to 1989 , he was a Research Scientist with General Network Corporation, New Haven, $\mathrm{CT}$, where he was involved with design and optimization of computer and telecommunications networks. He joined the University of Sydney, Sydney, Australia, in 1989 and became Professor of Imaging Science in 1997. He is currently a Professor of Computer Engineering at City University of Hong Kong. His research interests include image processing, pattern recognition, and bioinformatics. He is author or coauthor of one book and over 200 refereed technical papers in these areas.

Prof. Yan is a fellow of the International Association for Pattern Recognition, a fellow of the Institution of Engineers, Australia, and a member of the International Society for Computational Biology. 